\title{
SAMPLED-DATA REDESIGN FOR NONLINEAR MULTI-INPUT SYSTEMS
}

\author{
L. Grüne* and K. Worthmann \\ Mathematisches Institut, Fakultät für Mathematik, Physik und Informatik, Universität \\ Bayreuth, 95440 Bayreuth, Germany
}

\begin{abstract}
We investigate the sampled-data redesign problem for nonlinear control affine multi-input systems and consider sampled-data feedback laws for which the trajectories of the sampled-data closed loop system converge to the continuous time trajectories with a prescribed rate of convergence as sampling time vanishes. We analyze geometric conditions for the existence of such sampled-data feedback laws and give formulae and algorithms for their computation.
\end{abstract}

Keywords: sampled-data system, controller design, Lie brackets, Fliess expansion, Taylor expansion, nonlinear control affine system, convergence rate

\section{Introduction}

Feedback controllers are nowadays typically implemented using digital devices. In contrast to analog implementations, these devices are not able to evaluate the feedback law continuously in time but only at discrete sampling time instances. Thus, the controller must be designed as a sampleddata controller, whose simplest (and most widely used) implementation is a zero order hold, i.e., the feedback law is evaluated at each sampling time and the resulting control value is kept constant and applied on the sampling interval until the next sampling time.

A popular design method for sampled-data controllers is the design of a controller based on the continuous-time plant model, followed by a discretization of the controller. In other words, the continuous control function generated by the continuous-time controller is replaced by a piecewise constant and thus nonsmooth control function generated by the sampled-data controller. If the sampling interval is sufficiently small, then the choice of an appropriate sampled-data controller can be done in a very straightforward way, however, hardware or communication constraints may prohibit the use of small sampling intervals, in which case more sophisticated tech- 
niques have to be used. A good introduction to this subject in the nonlinear setting considered here can be found in the survey paper [11]. An important class of such techniques is the sampled-data redesign, in which a sampled-data controller is constructed which inherits certain properties of a previously designed feedback law for the continuous-time system. The survey papers $[4,12]$ summarize a couple of such redesign techniques. The analytical approaches in these papers are restricted to single-input systems, i.e., for systems with a one dimensional control variable, a condition which we relax in this paper.

More precisely, in this paper we extend the redesign technique presented in [5] to multi-input control affine nonlinear systems. This technique solves the redesign problem by designing a controller which is asymptotically optimal in the sense that we maximize the order at which the difference between the trajectories of the continuous and the sampled-data system converges to zero as the sampling time tends to zero. This amounts to investigating whether a sampled-data feedback law for a desired order exists and, in case the answer is positive, how it can be computed.

Concerning the conditions for the existence of higher order sampled-data feedback laws, it turns out that like in the single-input case the answer lies in the geometry of the system, expressed via the possible directions of the solution trajectories, which in turn are determined by the Lie brackets of the vector fields. Compared to the single-input case, the main difference of our multi-input results lies in the fact that the presence of more control variables typically facilitates the construction of a higher order sampleddata feedback law, an effect we illustrate in our numerical examples. In particular, it turns out that the design of sampled-data feedback laws of arbitrary order is always feasible if the control dimension equals the state dimension and the matrix composed of the control vector fields has full rank.

Since for higher orders the existence conditions and formulae for the sampled-data feedback laws become fairly complicated, we restrict our analytical results to low orders in order to illustrate the geometric nature of the conditions. For general orders we provide a Maple code which checks the respective conditions and computes the resulting sampled-data feedback, if possible. Here the second main difference to the single-input case appears: while in the single input case this computation was based on the successive solution of several one dimensional linear equations, the multi-input case can be tackled algorithmically via the solution of a suitable least squares problem. 


\section{Problem formulation}

We consider a nonlinear plant model

$$
\dot{x}(t)=f(x(t), u(t))
$$

with vector field $f: \mathbb{R}^{n} \times \mathbb{U} \rightarrow \mathbb{R}^{n}$ which is continuous and locally Lipschitz in $x$, state $x(t) \in \mathbb{R}^{n}$ and control $u(t) \in \mathbb{U} \subset \mathbb{R}^{m}$. Throughout the paper we assume that a smooth static state feedback $u_{0}: \mathbb{R}^{n} \rightarrow \mathbb{R}^{m}$ has been designed which solves some given control problem for the continuous-time closed-loop system

$$
\dot{x}(t)=f\left(x(t), u_{0}(x(t))\right) \quad x(0)=x_{0} .
$$

Our goal is now to design $u_{T}(x)$ such that the corresponding sampled-data solution $\phi_{T}\left(t, x_{0}, u_{T}\right)$ of the closed-loop system using a sampler and zero order hold

$$
\dot{x}(t)=f\left(x(t), u_{T}(x(k T))\right), \quad t \in[k T,(k+1) T)
$$

$k=0,1, \ldots$, reproduces the behavior of the continuous-time system and thus improves the performance of the sampled-data closed loop system.

Our approach uses an asymptotic analysis in order to study the difference between the continuous-time model (2) and the sampled-data model (3). To this end, for a function $a: \mathbb{R} \times \mathbb{R}^{n} \rightarrow \mathbb{R}$ we write $a(T, x)=\mathcal{O}\left(T^{q}\right)$, if for any compact set $K \subset \mathbb{R}^{n}$ there exists a constant $C>0$ (which may depend on $K)$ such that the inequality $a(T, x) \leq C T^{q}$ holds for all elements $x \in K$. If we consider a specific set $K$ we explicitly write $a(T, x)=\mathcal{O}\left(T^{q}\right)$ on $K$.

In order to obtain asymptotic estimates, we consider an "output" function $h: \mathbb{R}^{n} \rightarrow \mathbb{R}$ and derive series expansions for the difference

$$
\Delta h\left(T, x_{0}, u_{T}\right):=\left|h\left(\phi\left(T, x_{0}\right)\right)-h\left(\phi_{T}\left(T, x_{0}, u_{T}\right)\right)\right|,
$$

where $\phi\left(T, x_{0}\right)$ denotes the solution of the continuous-time system (2). Note that $h$ here is not a physical output of the system but rather a scalar auxiliary function which can be chosen arbitrarily. In particular, we will use $h_{i}(x)=x_{i}, i=1, \ldots, n$, in order to establish $\Delta h_{i}\left(T, x_{0}, u_{T}\right)=\mathcal{O}\left(T^{q}\right)$ which then implies

$$
\Delta \phi\left(T, x_{0}, u_{T}\right):=\left\|\phi\left(T, x_{0}\right)-\phi_{T}\left(T, x_{0}, u_{T}\right)\right\|_{\infty}=\mathcal{O}\left(T^{q}\right)
$$

measured in the maximum norm $\|\cdot\|_{\infty}$. From this estimate it follows by a standard induction argument that on each compact interval $\left[0, t^{*}\right]$ we obtain $\Delta \phi\left(t, x_{0}, u_{T}\right) \leq \mathcal{O}\left(T^{q-1}\right)$ for all times $t=k T, k \in \mathbb{N}$ with $t \in\left[0, t^{*}\right]$ 
which in particular allows to carry over stability properties from $\phi$ to $\phi_{T}$, see $[14,18]$.

In order to facilitate this analysis we restrict ourselves to control affine systems where the ordinary differential equations in (1)-(3) take the form

$$
\dot{x}(t)=g_{0}(x(t))+\sum_{i=1}^{m} g_{i}(x(t)) u_{0, i}(x(t)),
$$

with smooth vector fields $g_{0}, g_{1}, \ldots, g_{m}: \mathbb{R}^{n} \rightarrow \mathbb{R}^{n}$ and controls $u_{0,1}, \ldots, u_{0, m}: \mathbb{R}^{n} \rightarrow \mathbb{R}$. Note that the continuous-time feedback $u_{0}(x)=$ $\left(u_{0,1}(x), \ldots, u_{0, m}(x)\right)^{T}$ is represented in a vectorial form. In $[5,15]$ we investigated single input systems, i.e., $u(t) \in \mathbb{R}$. In this paper we extend these results to the multi-input case, i.e., $m>1$.

We look at sampled-data feedback laws meeting the following definition.

Definition 2.1. An admissible sampled-data feedback law $u_{T}$ is a family of maps $u_{T}: \mathbb{R}^{n} \rightarrow \mathbb{R}^{m}$, parameterized by the sampling period $T \in\left(0, T^{*}\right]$ for some maximal sampling period $T^{*}$, such that for each compact set $K \subset \mathbb{R}^{n}$ the inequality

$$
\sup _{x \in K, T \in\left(0, T^{*}\right]}\left\|u_{T}(x)\right\|_{\infty}<\infty
$$

holds.

Note that for existence and uniqueness of the solutions of (3), we do not need any continuity assumptions on $u_{T}$. Local boundedness is, however, imposed, because unbounded feedback laws are physically impossible to implement and often lead to closed-loop systems which are very sensitive to modelling or approximation errors, cf., e.g., the examples in $[3,14,16]$.

A special class of sampled-data feedback laws is given by

$$
u_{T}(x)=\sum_{j=0}^{M} T^{j} u_{j}(x),
$$

where the $u_{j}(x)$ are vectors $\left(u_{j, 1}(x), \ldots, u_{j, m}(x)\right)^{T}$. We will see later that this is exactly the form needed for our purpose. Inserting the sampled-data feedback (7) into our affine control system (6) leads to

$$
\begin{aligned}
\dot{x} & =g_{0}(x)+\sum_{i=1}^{m} g_{i}(x) u_{T, i}(x) \\
& =g_{0}(x)+\sum_{i=1}^{m} g_{i}(x) \sum_{j=0}^{M} T^{j} u_{j, i}(x) .
\end{aligned}
$$


Here the second index of $u_{j, i}(x)$ denotes the $i$-th component of the vector $u_{j}(x)$. Another way of writing $(8)$ is

$$
\dot{x}=g_{0}(x)+G(x) u_{T}(x) \quad \text { with } \quad G(x)=\left(\begin{array}{ccc}
g_{1,1}(x) & \cdots & g_{m, 1}(x) \\
\vdots & \ddots & \vdots \\
g_{1, n}(x) & \cdots & g_{m, n}(x)
\end{array}\right) .
$$

In the sequel we use the following notation: for subsets $D \subset \mathbb{R}^{n}$ we write $\operatorname{cl} D$, int $D$ for the closure and the interior of $D$. The notation $|\cdot|$ stands for the Euclidean norm while $\|x\|_{\infty}=\max _{i=1, \ldots, n}\left|x_{i}\right|$ denotes the maximum norm in $\mathbb{R}^{n}$. Furthermore, cf. [6], we denote the directional derivative of a function $h: \mathbb{R}^{n} \rightarrow \mathbb{R}$ in the direction of $g: \mathbb{R}^{n} \rightarrow \mathbb{R}^{n}$ by

$$
L_{g} h(x):=\frac{d}{d x} h(x) \cdot g(x)
$$

and the Lie bracket of vector fields $g_{i}, g_{j}: \mathbb{R}^{n} \rightarrow \mathbb{R}^{n}$ by

$$
\left[g_{i}, g_{j}\right]=\frac{d}{d x} g_{j} \cdot g_{i}-\frac{d}{d x} g_{i} \cdot g_{j}
$$

\section{Fliess series expansion}

In this section we provide the basic series expansion used for the redesign of $u_{T}$. Although the admissible sampled-data feedback $u_{T}$ according to Definition 2.1 may in principle be completely unrelated to $u_{0}=\left(u_{0,1}, \ldots, u_{0, m}\right)^{T}$, in the sequel it will turn out that a certain relation between $u_{0}$ and $u_{T}$ must hold. More precisely, we will see that the resulting sampled-data feedback (if existing) will be of the form (7) with $u_{0,1}(x), \ldots, u_{0, m}(x)$ from $(2)$ and $u_{1,1}(x), \ldots, u_{M, m}(x): \mathbb{R}^{n} \rightarrow \mathbb{R}$ being locally bounded functions. This structure appears to be rather natural and was also obtained as the outcome of the design procedure in several other papers, cf. [1,10,17] and also for our problem in the single input case [5]. Thus, we develop our series expansion for these feedback laws.

In order to formulate our result we define multinomial coefficients $\left(\begin{array}{c}n \\ n_{0} \ldots\end{array}\right):=\frac{n !}{n_{0} ! n_{1} ! \ldots n_{M} !}$ as well as multi-indices $\nu:=\left(n_{0}, n_{1}, \ldots, n_{M}\right)$ and use the notations $|\nu|:=n_{0}+n_{1}+\ldots+n_{M}$ and $\|\nu\|=\sum_{i=0}^{M} i n_{i}$. Our analytical considerations are based on the following theorem which is a generalization of $[15$, Theorem 3.1$]$ to the multi-input case.

Theorem 3.1. Consider the control affine system (6), a smooth function $h: \mathbb{R}^{n} \rightarrow \mathbb{R}$, the continuous-time closed-loop system (2) and the sampled- 
data closed-loop system (3) with controller $u_{T}$ given by (7). Then, for sufficiently small $T$, we can write:

$$
\begin{aligned}
h\left(\phi_{T}\left(T, x, u_{T}\right)\right) & =h(x)+\sum_{s=0}^{M} T^{s+1}\left[\sum_{i=1}^{m} L_{g_{i}} h(x) u_{s, i}+p_{s}\left(x, u_{0}, \ldots, u_{s-1}\right)\right] \\
& +\mathcal{O}\left(T^{M+2}\right)
\end{aligned}
$$

where $p_{0}(x)=L_{g_{0}} h(x)$ and $p_{s}\left(x, u_{0}, \ldots, u_{s-1}\right), s=1, \ldots, M$, is given by

$$
\begin{aligned}
\sum_{k=1}^{s} \sum_{i_{0}=0, \ldots, i_{k}=0}^{m} \frac{L_{g_{i 0}} \cdots L_{g_{i k}} h(x)}{(k+1) !} \\
\cdot \sum_{\substack{v \in \mathbb{N}_{0}^{m}: \\
\sum_{i=1}^{m} v_{i}=s-k}} \prod_{j=1}^{m}\left(\sum_{\substack{\left|\nu_{j}\right|=c_{j} \\
\left\|\nu_{j}\right\|=v_{j}}}\left(\begin{array}{c}
c_{j} \\
n_{0, j} n_{1, j} \cdots n_{M, j}
\end{array}\right) \prod_{l=0}^{M} u_{l, j}^{n_{l, j}}\right)
\end{aligned}
$$

with $u_{i}=\left(u_{i, 1}, \ldots, u_{i, m}\right)^{T}$. Here $c_{j}$ denotes $\#\left\{i_{l} \mid l=1, \ldots, k: i_{l}=j\right\}$.

For the proof, we need the following result, which can be found, e.g., in $[8$, Theorem 4.2$]$

Proposition 3.1. For $a_{i} \in \mathbb{R}, i=0,1,2, \ldots, M$ and $n \in \mathbb{N}$ we have the equality

$$
\left(a_{0}+a_{1}+\ldots+a_{M}\right)^{n}=\sum_{\substack{|\nu|=n \\
n_{0}, n_{1} \ldots n_{M} \geq 0}}\left(\begin{array}{c}
n \\
n_{0} \ldots n_{M}
\end{array}\right) a_{0}^{n_{0}} \cdots a_{M}^{n_{M}} .
$$

Proof. (Theorem 3.1) Using the Fliess series expansion, see [6, Theorem 3.1.5], we can write

$$
h(\phi(t, x))-h(x)=\sum_{k=0}^{\infty} \sum_{i_{0}=0, \ldots, i_{k}=0}^{m} L_{g_{i_{0}}} \ldots L_{g_{i_{k}}} h(x) \int_{0}^{t} d \xi_{i_{k}} \ldots d \xi_{i_{0}} .
$$

The expressions $\int_{0}^{t} d \xi_{i_{k}} \ldots d \xi_{i_{0}}$ denote iterated integrals as defined in [2]. Next we consider a single summand of the inner sum of this expression. We assign $i_{k}, \ldots, i_{0}$ to a vector $\left(c_{1}, \ldots, c_{m}\right)$, where $c_{j}:=\#\left\{i_{l} \mid l=0, \ldots, k\right.$ : $\left.i_{l}=j\right\}$. Since the values $u_{0}, \ldots, u_{m}$ are independent of $t$, the order of integration may be changed arbitrarily. That means that the value of the integral is independent of the order of the $L_{g_{i_{j}}}$-operators and it follows

$$
\int_{0}^{t} d \xi_{i_{k}} \ldots d \xi_{i_{0}}=\frac{T^{k+1}}{(k+1) !} \prod_{j=1}^{m} u_{T, j}^{c_{j}}
$$


Using Equation (12) we can write

$$
\frac{h\left(\phi_{T}\left(T, x, u_{T}\right)\right)-h(x)}{T}=\sum_{k=0}^{\infty} \sum_{i_{0}=0, \ldots, i_{k}=0}^{m} L_{g_{i_{0}}} \ldots L_{g_{i_{k}}} h(x) \frac{T^{k}}{(k+1) !} \prod_{j=1}^{m} u_{T, j}^{c_{j}} .
$$

Like in the single-input case [15] we use Lemma 3.1 in order to transform the components of (7). This leads to the expression

$$
u_{T, j}^{c_{j}}=\sum_{\substack{\left|\nu_{j}\right|=c_{j} \\
n_{0, j}, \ldots, n_{M, j} \geq 0}}\left(\begin{array}{c}
c_{j} \\
n_{0, j} n_{1, j} \cdots n_{M, j}
\end{array}\right) u_{0, j}^{n_{0, j}} \cdots u_{M, j}^{n_{M, j}} T^{\left\|\nu_{j}\right\|}
$$

with $\left|\nu_{j}\right|=\sum_{l=0}^{M} n_{l, j}$ and $\left\|\nu_{j}\right\|=\sum_{l=0}^{M} l n_{l, j}$. Hence it follows

$$
\begin{aligned}
& \frac{h\left(\phi_{T}\left(T, x, u_{T}\right)\right)-h(x)}{T}=H_{1}+\sum_{k=0}^{M} \sum_{i_{0}=0, \ldots, i_{k}=0}^{m} L_{g_{i_{0}}} \ldots L_{g_{i_{k}}} h(x) \frac{T^{k}}{(k+1) !} \\
& \cdot \prod_{j=1}^{m}\left(\sum_{\left|\nu_{j}\right|=c_{j}}\left(\begin{array}{c}
c_{j} \\
n_{0, j} n_{1, j} \cdots n_{M, j}
\end{array}\right) \prod_{l=0}^{M} u_{l, j}^{n_{l, j}} \cdot T^{\left\|\nu_{j}\right\|}\right),
\end{aligned}
$$

where $H_{1}$ denotes an $\mathcal{O}(M+1)$ term. Define $s:=k+\sum_{j=1}^{m}\left\|\nu_{j}\right\|=k+$ $\sum_{j=1}^{m} \sum_{l=0}^{M} l_{l, j}$ and sum over all terms of order $\leq M$. Collecting all terms of order strictly greater than $M$ in $H_{1}$ we can rewrite the last equation as

$$
\begin{gathered}
\frac{h\left(\phi_{T}\left(T, x, u_{T}\right)\right)-h(x)}{T}=H_{1}+\sum_{s=0}^{M} T^{s} \sum_{k=0}^{s} \sum_{i_{0}, \ldots, i_{k}=0}^{m} \frac{L_{g_{i_{0}}} \ldots L_{g_{i_{k}}} h(x)}{(k+1) !} \\
\cdot \sum_{\substack{v \in \mathbb{N}_{0}^{m}: \\
\sum_{i=1}^{m} v_{i}=s-k}} \prod_{j=1}^{m}\left(\sum_{\substack{\left|\nu_{j}\right|=c_{j} \\
\left\|\nu_{j}\right\|=v_{j}}}\left(\begin{array}{c}
c_{j} \\
n_{0, j} n_{1, j} \cdots n_{M, j}
\end{array}\right) \prod_{l=0}^{M} u_{l, j}^{n_{l, j}}\right) .
\end{gathered}
$$

Observe that for each $s>0$ the sum for $k=1 \ldots, s$ is exactly (11). Thus, in order to complete the proof it remains to show that the summands for $k=0$ equals the remaining terms in (10).

To this end first consider $s=0$ and $k=0$. This leads to $s-k=0$ and the only vector $v \in \mathbb{N}_{0}^{m}$ satisfying $\sum_{i=1}^{m} v_{i}=s-k$ is the zero vector. Consequently we obtain

$$
\sum_{i_{0}=0}^{m} L_{g_{i_{0}}} h(x) \underbrace{\sum_{0 \in \mathbb{N}_{0}^{m}} \prod_{j=1}^{m} \sum_{n_{0, j}=c_{j}} u_{0, j}^{n_{0, j}}}_{=\prod_{j=1}^{m} u_{0, j}^{c_{j}}}=L_{g_{0}} h(x)+\sum_{i=1}^{m} L_{g_{i}} h(x) u_{0, i} .
$$

For $s=1, \ldots, M$ and $k=0$ one computes $\sum_{i=1}^{m} v_{i}=s$ and differs between the cases $i_{0}=0$ and $i_{0}>0$. For $i_{0}=0$ this provides $L_{g_{0}} h(x)$ 
multiplied with a sum with respect to the empty set because it holds $c_{j}=0$ for all $j=1, \ldots, m$ and thus $\left|\nu_{j}\right|=0$ for all $j=1, \ldots, m$. This causes $\left\|\nu_{j}\right\|=0$ for all $j=1, \ldots, m$ and leads to a contradiction due to $\sum_{i=1}^{m}\left\|\nu_{j}\right\|=\sum_{i=1}^{m} v_{i}=s>0$. Accordingly $i_{0}=0$ doesn't provide any additional terms. Continuing we consider $i_{0}=i \in\{1, \ldots, m\}$. It holds $c_{i}=1$ and $c_{j}=0$ for all $j=1, \ldots, m$ with $j \neq i$. This leads to $\left|\nu_{j}\right|=0$ and $\left\|\nu_{j}\right\|=0$ for $j=1, \ldots, m$ with $j \neq i$. Consequently we have to consider $v=s e_{i}$ where $e_{i}$ denotes the i-th unit vector. Hence we multiply $L_{g_{i}} h(x)$ with $u_{s, i}$ due to

$$
c_{j}=\left\{\begin{array}{l}
1 \text { for } j=i \\
0 \text { otherwise }
\end{array}\right.
$$

Overall we obtain $\sum_{s=1}^{M} T^{s} \sum_{i=1}^{m} L_{g_{i}} h(x) u_{s, i}$ for $s>0$ and $k=0$, which finishes the proof.

Remark 3.1. While (10) is a straightforward extension of the single-input case, the $p_{s}$ differ in our multi-input case in terms of an additional combinatorial condition. One has to choose all vectors $v \in \mathbb{N}_{0}^{m}$ whose components add up to $s-k$.

Remark 3.2. For later reference we will explicitly compute the components of $p_{1}$ and $p_{2}$ according to (11). For $p_{1}\left(x, u_{0}\right)$ we have $s-k=0$ and thus the combinatorial condition $v \in \mathbb{N}_{0}^{m}: \sum_{i=1}^{m} v_{i}=s-k$ is only satisfied for $v=0_{\mathbb{N}_{0}^{m}}$. Now we have to distinguish three cases, the first one is $i_{0}=i_{1}=0$. Here $c_{j}=0$ for all $j \in\{1, \ldots, m\}$ and it results $\frac{1}{2} L_{g_{0}} L_{g_{0}} h(x)$. The second case is $i_{0}=0, i_{1} \neq 0$ and respectively $i_{0} \neq 0, i_{1}=0$. Here it exists exactly one $c_{j}>0$ and due to $\left\|\nu_{j}\right\|=0$ it follows

$$
\sum_{i=1}^{m} \frac{1}{2}\left(L_{g_{0}} L_{g_{i}} h(x)+L_{g_{i}} L_{g_{0}} h(x)\right) u_{0, i}(x) .
$$

The last case, $s=1, i_{0}>0, i_{1}>0$, provides

$$
\sum_{i=1, j=1}^{m} \frac{L_{g_{i}} L_{g_{j}} h(x)}{2} u_{0, j}(x) u_{0, i}(x) .
$$

For $p_{2}\left(x, u_{0}, u_{1}\right)$ we need to distinguish between $k=1$ and $k=2$. For $k=1$ we have $s-k=1$ and thus $v=e_{1}, \ldots, e_{m}$. Furthermore, we have to discern three cases. The first is $i_{0}=i_{1}=0$ which leads to the empty set. The second, $i_{0}=0, i_{1}>0$ respectively $i_{0}>0, i_{1}=0$, provides

$$
\sum_{i=1}^{m} \frac{1}{2}\left[L_{g_{0}} L_{g_{i}} h(x)+L_{g_{i}} L_{g_{0}} h(x)\right] u_{1, i}(x) .
$$


Finally, in the case $i_{0}>0$ and $i_{1}>0$ we obtain

$$
\sum_{\substack{i=1, j=1 \\ i \neq j}}^{m} \frac{1}{2} L_{g_{i}} L_{g_{j}} h(x) u_{1, i}(x) u_{0, j}(x)+\sum_{i=1}^{m} L_{g_{i}}^{2} h(x) u_{1, i}(x) u_{0, i}(x) .
$$

For $k=2$ it holds $s-k=0$ and thus we have only to regard $v=0_{\mathbb{N}_{0}^{m}}$. The computations take course analogously to the case $s=k=1$. Again we obtain terms dependent on components of $u_{0}$. Hence, altogether $p_{2}\left(x, u_{0}, u_{1}\right)$ provides

$$
\begin{aligned}
& +\frac{1}{2} \sum_{i=1}^{m}\left(\left[L_{g_{0}} L_{g_{i}}+L_{g_{i}} L_{g_{0}}\right] h(x)+\sum_{j=1}^{m} L_{g_{i}} L_{g_{j}} h(x) u_{0, j}(x)\right) u_{1, i}(x) \\
& +\frac{1}{6} \sum_{i=1}^{m}\left[L_{g_{i}} L_{g_{0}}^{2}+L_{g_{0}} L_{g_{i}} L_{g_{0}}+L_{g_{0}}^{2} L_{g_{i}}\right] h(x) u_{0, i}(x)+\frac{1}{6} L_{g_{0}}^{3} h(x) \\
& +\frac{1}{6} \sum_{i=1, j=1}^{m}\left[L_{g_{i}} L_{g_{j}} L_{g_{0}}+L_{g_{i}} L_{g_{0}} L_{g_{j}}+L_{g_{0}} L_{g_{i}} L_{g_{j}}\right] h(x) u_{0, i}(x) u_{0, j}(x) \\
& +\frac{1}{6} \sum_{i=1, j=1, k=1}^{m} L_{g_{i}} L_{g_{j}} L_{g_{k}} h(x) u_{0, i}(x) u_{0, j}(x) u_{0, k}(x)
\end{aligned}
$$

Remark 3.3. Computer algebra systems, such as Maple, can be used to compute expansions of the difference (4) for particular examples, cf. Remark 4.5 .

\section{Necessary and sufficient conditions}

In this section we investigate necessary and sufficient conditions for the existence of an admissible feedback law $u_{T}$ which achieves

$$
\Delta h\left(T, x, u_{T}\right)=\mathcal{O}\left(T^{q}\right)
$$

or

$$
\Delta \phi\left(T, x, u_{T}\right)=\mathcal{O}\left(T^{q}\right)
$$

and provide formulae for these feedback laws. Since the computations with respect to the sufficient condition turn out to be fairly involved we restrict our analytical computations to the case $q \leq 4$ and provide a Maple procedure for the general case. As we will see, $q=4$ is the first nontrivial case in the sense that that (15) and (16) for $q \leq 3$ can always be satisfied without any further conditions. For the case $q=4$ it turns out that the cases (15) and (16) require different conditions. In particular, for (16) we obtain a 
much stronger necessary condition than for (15). Thus, we state them in two separate theorems starting with (15).

The next theorem is a consequence from Theorem 3.1 performing a careful evaluation of the $p_{i}$-terms. It generalizes corresponding results for the single input-case $m=1$, see [15, Theorem 4.11] for the cases (i) and (ii), [5, Theorem 3.1] for case (iii), and also [4, Theorem 3.2]. For the formulation of the theorem we use the notation

$$
\begin{aligned}
u^{(i)}(x)=\left(\begin{array}{c}
u_{1}^{(i)}(x) \\
\vdots \\
u_{m}^{(i)}(x)
\end{array}\right) & =\left.\frac{1}{(i+1) !} \frac{d^{i}}{d t^{i}}\left(\begin{array}{c}
u_{0,1}(\phi(t, x)) \\
\vdots \\
u_{0, m}(\phi(t, x))
\end{array}\right)\right|_{t=0} \\
& =\left.\frac{1}{(i+1) !} \frac{d^{i} u_{0}(\phi(t, x))}{d t^{i}}\right|_{t=0} .
\end{aligned}
$$

Note that this definition coincides with the continuous-time controller for $i=0$. Furthermore we abbreviate

$$
\dot{u}_{j}(x):=\frac{\partial u_{0, j}(x)}{\partial x}\left[g_{0}+\sum_{i=1}^{m} g_{i} u_{0, i}\right]=2 u_{j}^{(1)}(x) .
$$

Theorem 4.1. Consider the vector field (6), the continuous-time closedloop system (2), the sampled-data closed-loop system (3), a smooth function $h: \mathbb{R}^{n} \rightarrow \mathbb{R}$ and a compact set $K \subset \mathbb{R}^{n}$. Then the following assertions hold for $u^{(i)}$ from $(17)$ :

(i) $\Delta h\left(T, x_{0}, u_{T}\right)=\mathcal{O}\left(T^{2}\right)$ holds on $K$ for

$$
u_{T}(x)=u^{(0)}(x) \text {. }
$$

(ii) $\Delta h\left(T, x_{0}, u_{T}\right)=\mathcal{O}\left(T^{3}\right)$ holds on $K$ for

$$
u_{T}(x)=u^{(0)}(x)+T u^{(1)}(x) .
$$

(iii) If there exists a bounded function $\alpha_{h}: K \rightarrow \mathbb{R}^{m}$ satisfying

$$
\sum_{i=1}^{m} L_{g_{i}} h(x) \alpha_{h, i}(x)=\sum_{i=1}^{m}\left[L_{\left[g_{0}, g_{i}\right]} h(x)+\sum_{\substack{j=1 \\ j \neq i}}^{m} L_{\left[g_{j}, g_{i}\right]} h(x) u_{0, j}(x)\right] \dot{u}_{i}(x),
$$

then there exists $u_{T}$ such that

$$
\Delta h\left(T, x_{0}, u_{T}\right)=\mathcal{O}\left(T^{4}\right)
$$

holds on $K$ with

$$
u_{T}(x)= \begin{cases}u^{(0)}(x)+T u^{(1)}(x)+T^{2} u^{(2)}(x)+\frac{T^{2}}{12} \alpha_{h}(x), & x \in \operatorname{cl} \widetilde{K} \\ u^{(0)}(x)+T u^{(1)}(x), & x \notin \operatorname{cl} \widetilde{K},\end{cases}
$$


where $\widetilde{K}:=\left\{x \in K \mid \exists i: L_{g_{i}} h(x) \neq 0\right\}$.

Conversely, if an admissible sampled-data feedback law $\tilde{u}_{T}=u_{T}+\mathcal{O}\left(T^{3}\right)$ for $u_{T}$ from (21) satisfies (20) on a set $\widehat{K} \subseteq \widetilde{K}$, then there exists a bounded function $\alpha$ satisfying (19) on $\mathrm{cl} \widehat{K}$.

Proof. Our smoothness assumptions enable us to use the Taylor series expansion for the solution of the ordinary differential equation (6). To this end we define the differential operator $\tilde{L}:=L_{g_{0}+\sum_{i=1}^{m} L_{g_{i}} u_{0, i}}$ and apply the Taylor series expansion to our output-function $h$, i.e.,

$$
h(\phi(T, x))-h(x)=\sum_{i=1}^{q-1} \frac{T^{i}}{i !} \tilde{L}^{i} h(x)+\mathcal{O}\left(T^{q}\right) .
$$

Hence, from the Taylor expansion of $h(\phi(t, x))$ in $t=0$ we obtain the identity

$$
\begin{aligned}
h(\phi(T, x)) & =h(x)+T \tilde{L} h(x)+\mathcal{O}\left(T^{2}\right) \\
& =h(x)+T\left[L_{g_{0}} h(x)+\sum_{i=1}^{m} L_{g_{i}} h(x) \cdot u_{0, i}(x)\right]+\mathcal{O}\left(T^{2}\right) \\
& =h(x)+T\left[\sum_{i=1}^{m} L_{g_{i}} h(x) u_{i}^{(0)}(x)+p_{0}(x)\right]+\mathcal{O}\left(T^{2}\right) \\
& =h\left(\phi_{T}\left(T, x, u_{T}\right)\right)+\mathcal{O}\left(T^{2}\right)
\end{aligned}
$$

using Theorem 3.1 in the last step. Thus (i) holds.

For the proof of (ii) we apply $\tilde{L}$ twice to the output-function $h$ and exploit the shape of $p_{1}\left(x, u_{0}\right)$ outlined in Remark 3.2:

$$
\begin{aligned}
h(\phi(t, x))= & h(x)+T \tilde{L} h(x)+\frac{T^{2}}{2} \tilde{L}^{2} h(x)+\mathcal{O}\left(T^{3}\right) \\
= & h(x)+T\left[\sum_{i=1}^{m} L_{g_{i}} h(x) u_{0, i}(x)+p_{0}(x)\right]+T^{2} p_{1}\left(x, u_{0}\right) \\
& +\frac{T^{2}}{2} \sum_{i=1}^{m} L_{g_{i}} h(x) \frac{\partial u_{0, i}(x)}{\partial x}\left[g_{0}+\sum_{i=1}^{m} g_{i} u_{0, i}\right]+\mathcal{O}\left(T^{3}\right) \\
= & h(x)+\sum_{s=0}^{1} T^{s+1}\left[\sum_{i=1}^{m} L_{g_{i}} h(x) u_{i}^{(s)}(x)+p_{s}\left(x, u^{(0)}, \ldots, u^{(s-1)}\right)\right] \\
& +\mathcal{O}\left(T^{3}\right) \\
= & h\left(\phi_{T}\left(T, x, u_{T}\right)\right)+\mathcal{O}\left(T^{3}\right)
\end{aligned}
$$


where we used Theorem 3.1 and $u_{T}=u^{(0)}+T u^{(1)}$ in the last step. This shows (ii).

In order to prove (iii) we have to examine the Taylor series expansion up to order four. To this aim we consider the threefold application of $\tilde{L}$ and use the identity $\dot{u}_{j}(x)=\tilde{L} u_{0, j}(x)$ :

$$
\begin{aligned}
\tilde{L}^{3} h(x) & =L_{g_{0}}^{3} h(x)+\sum_{i=1}^{m}\left[L_{g_{i}} L_{g_{0}}^{2}+L_{g_{0}} L_{g_{i}} L_{g_{0}}+L_{g_{0}}^{2} L_{g_{i}}\right] h(x) u_{0, i}(x)+ \\
& +\sum_{j=1}^{m} \sum_{i=1}^{m}\left[L_{g_{j}} L_{g_{i}} L_{g_{0}}+L_{g_{j}} L_{g_{0}} L_{g_{i}}+L_{g_{0}} L_{g_{j}} L_{g_{i}}\right] h(x) u_{0, i}(x) u_{0, j}(x) \\
& +\sum_{i, j, k=1}^{m} L_{g_{k}} L_{g_{j}} L_{g_{i}} h(x) u_{0, i}(x) u_{0, j}(x) u_{0, k}(x)+ \\
& +\sum_{i=1}^{m}\left[L_{g_{i}} L_{g_{0}}+2 L_{g_{0}} L_{g_{i}}\right] h(x) \dot{u}_{i}(x)+\sum_{i=1}^{m} L_{g_{i}} h(x) \tilde{L} \dot{u}_{i}(x)+ \\
& +\sum_{i=1, j=1}^{m} L_{g_{i}} L_{g_{j}} h(x)\left[2 \dot{u}_{j}(x) u_{0, i}(x)+\dot{u}_{i}(x) u_{0, j}(x)\right] .
\end{aligned}
$$

A comparison between (27) and the terms resulting from (14) using $u_{T}$ from (21) yields

$$
\begin{aligned}
& h(\phi(T, x))= \sum_{s=0}^{2} T^{s+1}\left[\sum_{i=1}^{m} L_{g_{i}} h(x) u_{i}^{(s)}(x)+p_{s}\left(x, u^{(0)}, \ldots, u^{(s-1)}\right)\right] \\
&+\frac{1}{12} \sum_{i=1}^{m}\left[L_{g_{0}} L_{g_{i}} h(x)+\sum_{\substack{j=1 \\
j \neq i}}^{m} L_{g_{j}} L_{g_{i}} h(x) u_{0, j}(x)-\right. \\
&\left.\quad-L_{g_{i}} L_{g_{0}} h(x)-\sum_{\substack{j=1 \\
j \neq i}}^{m} L_{g_{i}} L_{g_{j}} h(x) u_{0, j}(x)\right] \dot{u}_{i}(x) \\
&+h(x)+\mathcal{O}\left(T^{4}\right) .
\end{aligned}
$$


With $L_{g_{0}} L_{g_{i}} h(x)-L_{g_{i}} L_{g_{0}} h(x)=L_{\left[g_{0}, g_{i}\right]} h(x)$ one obtains

$$
\begin{aligned}
h(\phi(T, x)) & =\sum_{s=0}^{2} T^{s+1}\left[\sum_{i=1}^{m} L_{g_{i}} h(x) u_{i}^{(s)}(x)+p_{s}\left(x, u^{(0)}, \ldots, u^{(s-1)}\right)\right] \\
& +\frac{1}{12} \sum_{i=1}^{m}\left[L_{\left[g_{0}, g_{i}\right]} h(x)+\sum_{\substack{j=1 \\
j \neq i}}^{m} L_{\left[g_{j}, g_{i}\right]} h(x) u_{0, j}(x)\right] \dot{u}_{i}(x) \\
& +h(x)+\mathcal{O}\left(T^{4}\right)=h\left(\phi_{T}\left(T, x, u_{T}\right)\right)+\mathcal{O}\left(T^{4}\right),
\end{aligned}
$$

where we used Theorem 3.1 and the definition of $u_{T}$ and $\alpha_{h}$ in the last step. Thus, the choice (21) ensures a forth order approximation. Note that the function $\alpha_{h}$ is bounded on $K$ by assumption, which in particular implies that the control law (21) is admissible in the sense of Definition 2.1 on $\widetilde{K}$.

For the converse statement, if $u_{T}$ from (21) satisfies (20) on $\widehat{K} \subseteq \widetilde{K}$ and Definition 2.1, then the function $\alpha$ in (21) must be bounded. Hence, for each boundary point $x \in \partial \widehat{K}$ we can find a sequence $x_{k} \rightarrow x$ such that $\alpha\left(x_{k}\right)$ is convergent and define $\alpha(x)=\lim _{k \rightarrow \infty} \alpha\left(x_{k}\right)$. Then, since all coefficients in (19) are continuous, we obtain that (19) also holds for $x \in \partial \widehat{K}$, i.e., on cl $\widehat{K}$ and the boundedness follows immediately.

Remark 4.1. Note that the converse part of statement (iii) is rather weak, as it only provides a necessary condition for the existence of feedback laws of the specific form (21) but not for arbitrary admissible sampled-data feedback laws satisfying (20). It is however, an important building block for the much stronger necessary condition for $\Delta \phi\left(T, x, u_{T}\right)=\mathcal{O}\left(T^{4}\right)$ given in Theorem 4.2, below.

Remark 4.2. In (21) we distinguish between $x \in \operatorname{cl} \widetilde{K}$ and $x \notin \operatorname{cl} \widetilde{K}$. This case differentiation can be interpreted in terms of relative degree (see [7] for a definition and [12] for the role of the relative degree in sampled-data feedback design). System (6) with output function $h$ has relative degree one on $\mathrm{cl} \widetilde{K}$ while the relative degree is strictly larger on $K \backslash \mathrm{cl} \widetilde{K}$. This explains why the feedback law (21) has different structure inside and outside cl $\widetilde{K}$.

Remark 4.3. For driftless systems, i.e., $g_{0}(x) \equiv 0$, the lie-bracket $\left[g_{0}, g_{i}\right]$ in (19) is equal to zero for all $i=1, \ldots, m$. Hence, condition (19) is always satisfied for $m=1$ and easier to evaluate otherwise.

Now we turn to (16) and deduce assertions for the full state trajectory from Theorem 4.1 by choosing $h_{k}(x)=x_{k}, k=1, \ldots, n$. 
Theorem 4.2. Consider the control affine system (6), the continuous-time closed-loop system (2), the sampled-data closed-loop system (3) and a compact set $K \subset \mathbb{R}^{n}$ satisfying $K=\mathrm{cl}$ int $K$. Then the following assertions hold for $u^{(i)}$ from $(17)$ :

(i) $\Delta \phi\left(T, x_{0}, u_{T}\right)=\mathcal{O}\left(T^{2}\right)$ holds on $K$ for

$$
u_{T}(x)=u^{(0)}(x) .
$$

(ii) $\Delta \phi\left(T, x_{0}, u_{T}\right)=\mathcal{O}\left(T^{3}\right)$ holds on $K$ for

$$
u_{T}(x)=u^{(0)}(x)+T u^{(1)}(x) .
$$

(iii) If there exists a bounded function $\alpha: K \rightarrow \mathbb{R}^{m}$ satisfying

$$
\sum_{i=1}^{m}\left[\left[g_{0}, g_{i}\right](x)+\sum_{\substack{j=1 \\ j \neq i}}^{m}\left[g_{j}, g_{i}\right](x) u_{0, j}(x)\right] \dot{u}_{i}(x)=\sum_{i=1}^{m} \alpha_{i}(x) g_{i}(x)
$$

then

$$
\Delta \phi\left(T, x_{0}, u_{T}\right)=\mathcal{O}\left(T^{4}\right)
$$

holds on $K$ for

$$
u_{T}(x)= \begin{cases}u^{(0)}(x)+T u^{(1)}(x)+T^{2} u^{(2)}(x)+\frac{T^{2}}{12} \alpha(x), & x \in \operatorname{cl} \widetilde{K} \\ \text { arbitrary, } & x \notin \operatorname{cl} \widetilde{K}\end{cases}
$$

with $\widetilde{K}:=\left\{x \in K \mid \exists i: g_{i}(x) \neq 0\right\}$. Furthermore, on

$$
K^{*}=\{x \in K \mid G(x) \text { from (9) has full column rank }\} \text {, }
$$

any feedback law $\tilde{u}_{T}$ satisfying $\Delta \phi\left(T, x_{0}, \tilde{u}_{T}\right)=\mathcal{O}\left(T^{q}\right), q=2,3,4$, is of the form $\tilde{u}_{T}(x)=u_{T}(x)+\mathcal{O}\left(T^{q-1}\right)$ for $u_{T}$ from (i), (ii) or (iii), respectively, and the function $\alpha$ in (29) is unique if it exists. On $\mathrm{cl} K^{*}$ the sufficient condition (29) is also necessary for the existence of $u_{T}$ in (iii).

Proof. First note that (16) is equivalent to (15) for $h_{i}(x)=x_{i}, i=1, \ldots, n$. Hence, assertions (i) and (ii) follow immediately from Theorem 4.1 applied to $h_{i}(x)=x_{i}, i=1, \ldots, n$.

For the proof of (iii), we first show that under condition (29) any feedback of the form (30) satisfies the assertion.

First note that for $x \notin \mathrm{cl} \widetilde{K}$ the feedback value $u_{T}(x)$ is indeed arbitrary. This follows since on $K \backslash \operatorname{cl} \widetilde{K}$ the control system is given by $\dot{x}=g_{0}(x)$. Thus, on the open set int $(K \backslash \operatorname{cl} \widetilde{K})$ the Taylor series expansions of $\phi(t, x)$ and $\phi_{T}\left(t, x, u_{T}\right)$ coincide for any order, regardless of the values of $u_{0}$ and $u_{T}$, i.e., we obtain (16) for any $M>0$ for arbitrary $u_{T}$. By continuity of 
the expressions in the Taylor series expansion this property carries over to $\operatorname{cl} \operatorname{int}(K \backslash \operatorname{cl} \widetilde{K})$ which contains $K \backslash \operatorname{cl} \widetilde{K}$ because we have assumed $K=$ clint $K$.

It is, hence, sufficient to show that $u_{T}$ satisfies the assertion for $x \in \operatorname{cl} \widetilde{K}$. Assume that the function $\alpha$ exists and is bounded. Fix $i \in\{1, \ldots, n\}$ and consider the function $h_{i}(x)=x_{i}$. A simple computation using the identities

$$
L_{g_{j}} h_{i}(x)=g_{j, i}(x) \quad \text { and } \quad L_{\left[g_{k}, g_{j}\right]} h_{i}(x)=\left[g_{k}, g_{j}\right](x)_{i}
$$

shows that the function $\alpha$ from (29) satisfies

$$
\begin{aligned}
\sum_{i=1}^{m} \alpha_{i}(x) g_{i}(x) & =\sum_{i=1}^{m}\left[\left[g_{0}, g_{i}\right](x)+\sum_{\substack{j=1 \\
j \neq i}}^{m}\left[g_{j}, g_{i}\right](x) u_{0, j}(x)\right] \dot{u}_{i}(x) \\
& =\sum_{i=1}^{m}\left[L_{\left[g_{0}, g_{i}\right]} h(x)+\sum_{\substack{j=1 \\
j \neq i}}^{m} L_{\left[g_{j}, g_{i}\right]} h(x) u_{0, j}(x)\right] \dot{u}_{i}(x) .
\end{aligned}
$$

Thus, the feedback is of the form (21) for $h=h_{i}$ and we can use Theorem 4.1 to conclude (15) for $q=4$ and $i=1, \ldots, n$ and thus (16).

Now we show the claimed form of the $\tilde{u}_{T}$ on $K^{*}$ : From Theorem 3.1 for $M=0$ it follows that any $\tilde{u}_{T}$ satisfying $\Delta \phi\left(T, x_{0}, \tilde{u}_{T}\right)=\mathcal{O}\left(T^{2}\right)$ must fulfill

$$
\sum_{i=1}^{m} L_{g_{i}} h_{k}(x) u_{0, i}(x)=\sum_{i=1}^{m} L_{g_{i}} h_{k}(x) \tilde{u}_{T, i}(x)+\mathcal{O}(T)
$$

for $k=1, \ldots, n$ in order to get the equality " $(23)=(24)$ " (for $\tilde{u}_{T}$ instead of $u_{T}$ ) for all $h_{k}$. Using again $L_{g_{j}} h_{i}(x)=g_{j, i}(x)$ one sees that this is equivalent to

$$
G(x) u_{0}(x)=G(x) \tilde{u}_{T}(x)+\mathcal{O}(T)
$$

and since $G(x)$ has full column rank this implies $\tilde{u}_{T}(x)=u_{0}+\mathcal{O}(T)$. The statements for (ii) and (iii) now follow analogously by induction using the equalities " $(25)=(26)$ " and (28). The uniqueness of $\alpha$ on $K^{*}$ follows again from the full column rank of $G(x)$ because the right hand side of (29) equals $G(x) \alpha(x)$.

Finally, using the uniqueness of $u_{T}$ in (iii) up to higher order terms, the necessity of $(29)$ on $\mathrm{cl} K^{*}$ follows from the converse statement in Theorem 4.1(iii) for $\widehat{K}=K^{*}$.

Remark 4.4. Theorem 4.2 has a nice geometric interpretation if we consider the possible directions of the system trajectories. To this end, consider 
the expansions

$$
\begin{aligned}
\phi(T, x) & =v_{0}+T v_{1}+T^{2} v_{2}+T^{3} v_{3}+\ldots \\
\phi_{T}\left(T, x, u_{T}\right) & =w_{0}+T w_{1}+T^{2} w_{2}+T^{3} w_{3}+\ldots
\end{aligned}
$$

in which the vectors $v_{i}$ and $w_{i}$ determine the directions of the respective solution trajectories. While the control value in $\phi$ may vary in time, the control value in $\phi_{T}$ is constant on the sampling interval $[0, T)$. Thus, for each $i=0,1, \ldots$ the set of possible directions $v_{i}$ which can be generated by different choices of $u_{0}$ is larger or equal than the corresponding set of possible directions $w_{i}$ generated by different $u_{T}$.

The cases (i) and (ii) now show that the sets of possible directions $v_{i}$ and $w_{i}$ are indeed identical for $i=0,1$ and 2, because (i) and (ii) are unconditionally feasible provided $u_{T}$ is chosen appropriately. Note that the $T$-dependence of $u_{T}$ is crucial in (ii) because it gives us the additional flexibility needed for achieving $w_{2}=v_{2}$.

This is no longer possible for the directions $v_{3}$ and $w_{3}$ which affect the trajectories with order $\mathcal{O}\left(T^{3}\right)$. Indeed, our analysis shows that the direction $v_{3}$ can be decomposed as $v_{3}=v_{3}^{1}+v_{3}^{2}$, such that $w_{3}=v_{3}^{1}$ can always be achieved via the $u^{(2)}$ term in $u_{T}$ while $v_{3}^{2}$ cannot in general be reproduced by $w_{3}$. This direction $v_{3}^{2}$ is exactly the expression appearing on the left hand side of (29) which depends on the Lie brackets of the vector fields and on the continuous-time feedback law $u_{0}$. Condition (29) now demands that $v_{3}^{2}$ lies in $\operatorname{span}\left\langle g_{1}, \ldots, g_{m}\right\rangle$ such that it can be compensated by the $\alpha$-term of the sampled-data feedback law $u_{T}$.

Remark 4.5. While the formulation of condition (29) is suitable for the geometric interpretation, it is difficult to generalize it to orders $\mathcal{O}\left(T^{q}\right), q \geq 5$. However, using Theorem 3.1 directly we can obtain a simple recursive procedure for computing $u_{T}$ for arbitrary orders: Assuming that $u_{0}, \ldots, u_{M-1}$ in (7) are already determined and realize the order $\mathcal{O}\left(T^{M+1}\right)$. Then, comparing the summand for $s=M$ in (10) with the summand for $s=M$ in the Taylor expansion of $\phi(T, x)$ leads to a (in general overdetermined) linear system

$$
G(x) u_{M}(x)=b(x) .
$$

If (31) admits a solution, then this defines the $M$-th component of $u_{T}$ in (7) which then realizes the order $\mathcal{O}\left(T^{M+2}\right)$. If (31) does not admit a solution, then the order $\mathcal{O}\left(T^{M+2}\right)$ cannot be achieved by a sampled data feedback law. 
This procedure can be efficiently implemented in MAPLE using the least squares solver in order to solve (31) and checking the residual in order to decide whether (31) is solvable. The MAPLE implementation is available on www.math.uni-bayreuth.de/ lgruene/publ/redesign_multiinput.html.

Furhermore, this procedure shows that we can always achieve any desired order if the matrix $G$ is square, i.e., the control dimension $m$ equals the space dimension $n$, and invertible.

Remark 4.6. In [13] it was shown for single-input systems, i.e., $m=1$, that the condition $\left[g_{0}, g_{1}\right] \in \operatorname{span}\left\langle g_{1}\right\rangle$ is necessary and sufficient for the existence of sampled-data feedback laws $u_{T}$ realising $\Delta \phi(T, x)=\mathcal{O}\left(T^{q}\right)$ for all $q \geq 2$ and all continuous-time feedback laws $u_{0}$. We conjecture that the generalization of this condition to the multi-input case is $\left[g_{i}, g_{j}\right] \in \operatorname{span}\left\langle g_{1}, \ldots, g_{m}\right\rangle$ for all $i, j=0, \ldots, m$. Note that the sampleddata feedback laws considered in [13] are not necessarily locally bounded and thus may not fulfill our Definition 2.1.

\section{Examples}

We illustrate our results by two examples. We first consider the second order version of the Moore-Greitzer jet engine model

$$
\left(\begin{array}{l}
\dot{x}_{1} \\
\dot{x}_{2}
\end{array}\right)=\left(\begin{array}{c}
-x_{2}-3 x_{1}^{2} / 2-x_{1}^{3} / 2 \\
0
\end{array}\right)+\left(\begin{array}{c}
0 \\
-1
\end{array}\right) u_{0,1}
$$

with the continuous-time stabilising backstepping feedback law $u_{0,1}(x)=$ $-7 x_{1}+5 x_{2}$ derived in [9, Section 2.4.3]. Here the condition (29) shows that no admissible sampled-data feedback $u_{T}$ satisfies $\Delta \phi\left(T, x, u_{T}\right) \leq \mathcal{O}\left(T^{4}\right)$, cf., [5, Section 4]. Now we examine this system with an additional control $u_{0,2} \equiv 0$, i.e.,

$$
\left(\begin{array}{l}
\dot{x}_{1} \\
\dot{x}_{2}
\end{array}\right)=\left(\begin{array}{c}
-x_{2}-3 x_{1}^{2} / 2-x_{1}^{3} / 2 \\
0
\end{array}\right)-\left(\begin{array}{l}
0 \\
1
\end{array}\right) u_{0,1}+\left(\begin{array}{c}
g_{2,1}(x) \\
g_{2,2}(x)
\end{array}\right) u_{0,2} .
$$

Note that this is now an academical example because the vector field $g_{2}=\left(g_{2,1}, g_{2,2}\right)^{T}$ and its control $u_{0,2}$ do not have any physical meaning. Nevertheless, the additional input allows for the design of higher order sampled-data feedback laws. Indeed, while $u_{0,2} \equiv 0$ implies that the left hand side of our condition (29) coincides for (32) and (33) and evaluates to

$$
\left(\begin{array}{c}
-1 \\
0
\end{array}\right) \dot{u}_{1}(x)=\left(\begin{array}{c}
-35 x_{1}+18 x_{2}-\frac{21}{2} x_{1}^{2}-\frac{7}{2} x_{1}^{3} \\
0
\end{array}\right)
$$


with $\dot{u}_{1}(x)$ from (18), on the right hand side of condition (29) the coefficients of $g_{2}$ yield additional degrees of freedom and (29) becomes

$$
\left(\begin{array}{c}
-1 \\
0
\end{array}\right) \dot{u}_{1}(x)=\left(\begin{array}{c}
\alpha_{2}(x) g_{2,1}(x) \\
-\alpha_{1}(x)+\alpha_{2}(x) g_{2,2}(x)
\end{array}\right)
$$

It is easily seen that this equality is satisfied, e.g., for $g_{2}(x)=(1,0)^{T}$ and $\alpha(x)=\left(0,-\dot{u}_{1}(x)\right)^{T}$. The performance for this choice of $g_{2}$ and $\alpha$ and the resulting feedback law

$$
u_{T}(x)=u^{(0)}(x)+T u^{(1)}(x)+T^{2} u^{(2)}(x)+\frac{T}{12} \alpha(x)
$$

is shown in Figure 1.
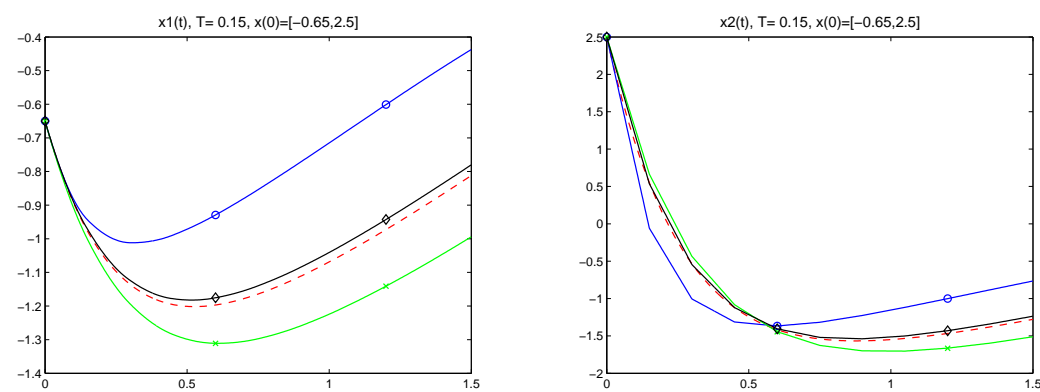

Fig. 1. $x(t)$ for example (33), $T=0.15$. Continuous-time solution (- $)$; sampled-data solution for order $q=2(\mathrm{o}), q=3(\mathrm{x})$ and $q=4(\diamond)$.

By means of our Maple-procedure, we may compute feedbacks of even higher order. We took this approach to compute the trajectory for $q=6$ in Figure 2. Remarkable is that neither the sampled-data feedback for $q=2$ (i.e., $u_{T}=u_{0}$ ) nor the feedback for $q=3$ preserve the asymptotic stability of the continuous-time system. In contrast to that the fourth order feedback preserves asymptotic stability and the feedback for $q=6$ provides an even better performance despite the large sampling period.

For further investigation of our analytically constructed control laws we analyze the three dimensional Moore-Greitzer model. Adding an additional 

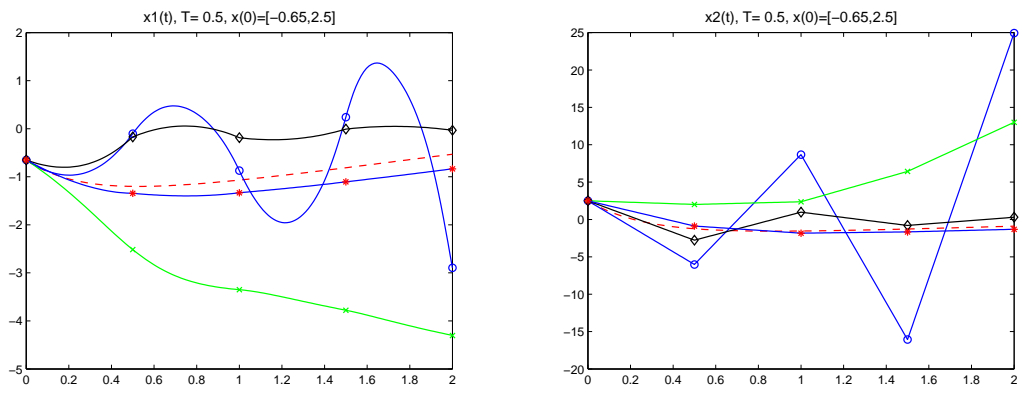

Fig. 2. $x(t)$ for example (33), $T=0.5$. Continuous-time solution (--); sampled-data solution for order $q=2(\mathrm{o}), q=3(\mathrm{x}), q=4(\diamond)$ and $q=6\left(^{*}\right)$.

control input $u_{0,2} \equiv 0$ analogously to (33) we obtain the system

$$
\left(\begin{array}{c}
\dot{x}_{1} \\
\dot{x}_{2} \\
\dot{x}_{3}
\end{array}\right)=\left(\begin{array}{c}
-x_{2}-\frac{3}{2} x_{1}^{2}-\frac{1}{2} x_{1}^{3}-3 x_{3} x_{1}-3 x_{3} \\
0 \\
-\sigma x_{3}\left(x_{3}+2 x_{1}+x_{1}^{2}\right)
\end{array}\right)+\left(\begin{array}{c}
0 \\
-1 \\
0
\end{array}\right) u_{0,1}+\left(\begin{array}{l}
1 \\
0 \\
0
\end{array}\right) u_{0,2}
$$

with $\sigma=2$ and continuous-time controller (cf. [9, Section 2.4.2])

$$
\begin{aligned}
u_{0,1}(x)= & -\left(c_{1}-3 x_{1}\right)\left(-x_{2}-\frac{3}{2} x_{1}^{2}-\frac{1}{2} x_{1}^{3}-3 x_{1} x_{3}-3 x_{3}\right) \\
& +c_{2}\left(x_{2}-c_{1} x_{1}+\frac{3}{2} x_{1}^{2}+3 x_{3}\right)-x_{1}-3 \sigma x_{3}\left(x_{3}+2 x_{1}+x_{1}^{2}\right) \\
u_{0,2}(x)= & 0
\end{aligned}
$$

using the parameters $c_{1}=1$ and $c_{2}=50$. Again, the Maple-routine provides sampled-data feedbacks for $q=4,5$, but reveals that there does not exist a control law for order $q=6$. However, the order $q=6$ becomes feasible if one adds $g_{3}(x)=(0,0,1)^{T} u_{0,3}(x)$ with $u_{0,3} \equiv 0$ as a second additional control term. Figure 3 presents the numerical simulations of this design procedure.

The sampled continuous-time feedback $u_{T}=u_{0}$ does not retain the asymptotic stability of the continuous-time solution. Instead, it exhibits an asymptotically stable periodic trajectory and even divergence for sampling periods $T \geq 0.052$. In contrast to that $u_{T}$ for $q=4$ and $q=6$ preserve the asymptotic stability for $T \leq 0.05$ for $q=4$ and $T \leq 0.064$ for $q=6$, respectively, while for larger sampling intervals the solutions become first periodic and eventually divergent, too. 

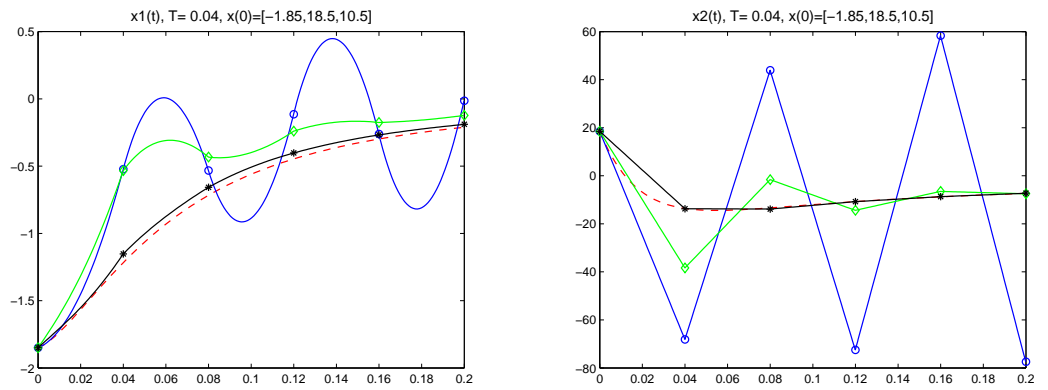

Fig. 3. $x(t)$ for example (36), $T=0.04$. Continuous-time solution (- $)$; sampled-data solution for orders $q=2(\mathrm{o}), q=4(\diamond)$ and $q=6(*)$.

\section{References}

1. A. Arapostathis, B. Jakubczyk, H.-G. Lee, S. Marcus, and E. D. SonTAG, The effect of sampling on linear equivalence and feedback linearization, Syst. Contr. Lett., 13 (1989), pp. 373-381.

2. L. GRÜNE AND P. E. KLOEDEN, Higher order numerical schemes for affinely controlled nonlinear systems, Numer. Math., 89 (2001), pp. 669-690.

3. L. GRÜNE AND D. NEŠÍc, Optimization based stabilization of sampled-data nonlinear systems via their approximate discrete-time models, SIAM J. Control Optim., 42 (2003), pp. 98-122.

4. L. Grüne, D. Nešić, J. Pannek, And K. Worthmann, Redesign techniques for nonlinear sampled-data systems, at-Automatisierungstechnik. submitted.

5. L. Grüne, D. NEŠIĆ, AND K. Worthmann, Continuous-time controller redesign for digital implementation: a trajectory based approach, Automatica. provisionally accepted.

6. A. IsIDORI, Nonlinear control systems, Prentice Hall, Upper Saddle River, New Jersey, 3rd ed., 2002.

7. H. K. Khalil, Nonlinear systems, Prentice Hall, Upper Saddle River, New Jersey, 3rd ed., 2002.

8. V. Krishnamurthy, Combinatorics: theory and applications, Affiliated EastWest Press, Madras, 1985.

9. M. Krstić, I. Kanellakopoulos, and P. V. Kokotović, Nonlinear and adaptive control design, John Wiley \& Sons, New York, 1995.

10. D. S. LAILA AND D. NEŠIĆ, Changing supply rates for input-output to state stable discrete-time nonlinear systems with applications, Automatica, 39 (2003), pp. 821-835.

11. D. S. Laila, D. NeŠIĆ, AND A. Astolfi, Sampled-data control of nonlinear systems, in Advanced Topics in Control Systems Theory: Lecture Notes from FAP 2005, A. Loría, F. Lamnabhi-Lagarrigue, and E. Panteley, eds., vol. 328 of Lecture Notes in Control and Information Sciences, Berlin, Heidelberg, 2006, Springer-Verlag, pp. 91-137. 
12. S. Monaco And D. Normand-Cyrot, Issues on nonlinear digital control, Europ. J. Control, 7 (2001), pp. 160-178.

13. S. Monaco And D. Normand-Cyrot, Input-state matching under digital control, in Proc. 45th IEEE CDC, San Diego, 2006.

14. D. NešIć, A. R. TeEL, And P. V. Kokotović, Sufficient conditions for stabilization of sampled-data nonlinear systems via discrete-time approximations, Syst. Contr. Lett., 38 (1999), pp. 259-270.

15. D. NeŠIĆ AND L. GRÜNE, Lyapunov based continuous-time nonlinear controller redesign for sampled-data implementation, Automatica, 41 (2005), pp. $1143-1156$.

16. D. NEŠIĆ AND A. R. TEEL, A framework for stabilization of nonlinear sampled-data systems based on their approximate discrete-time models, IEEE Trans. Automat. Control, 49 (7) (2004), pp. 1103-1122.

17. D. NešIĆ AND A. R. TEEL, Stabilization of sampled-data nonlinear systems via backstepping on their euler approximate model, Automatica, 42 (2006), pp. 1801-1808.

18. D. NešIĆ, A. R. Teel, And E. D. Sontag, Formulas relating KL stability estimates of discrete-time and sampled-data nonlinear systems, Syst. Contr. Lett., 38 (1999), pp. 49-60. 